\title{
BMJ Open Prevalence of multimorbidity in the adult population attending primary care in Portugal: a cross-sectional study
}

\author{
Filipe Prazeres, ${ }^{1,2}$ Luiz Santiago ${ }^{1,3}$
}

To cite: Prazeres F,

Santiago L. Prevalence of multimorbidity in the adult population attending primary care in Portugal: a crosssectional study. BMJ Open 2015;5:e009287.

doi:10.1136/bmjopen-2015009287

- Prepublication history for this paper is available online To view these files please visit the journal online (http://dx.doi.org/10.1136/ bmjopen-2015-009287)

Received 1 July 2015 Revised 25 August 2015 Accepted 2 September 2015

CrossMark

${ }^{1}$ Faculdade de Ciências da Saúde, Universidade da Beira Interior, Covilhã, Portugal ${ }^{2}$ Centro de Saúde de Aveiro, Aveiro, Portugal

${ }^{3}$ USF Topázio, Coimbra, Portugal

Correspondence to Dr Filipe Prazeres;

filipeprazeresmd@gmail.com

\section{ABSTRACT}

Objectives: To determine the prevalence of multimorbidity in the adult population attending primary care in Portugal, to identify associated sociodemographic factors, and to reveal combinations of chronic health problems.

Design: Cross-sectional, analytical study.

Setting: Primary Care Centres in mainland Portugal across the five Portuguese Healthcare Administrative Regions.

Participants: 1279 women and 714 men agreed to participate. The mean age was 56.3 years (59.0 years for men; 54.8 years for women). The most frequent marital status was married/cohabiting $(69.5 \%)$. The most predominant living arrangement was living as a couple $(57.2 \%)$. A considerable proportion consisted of pensioners/retirees (41.5\%) and adults with a low educational level $(48.7 \%)$. Sufficient monthly income was reported in $54.4 \%$ of the cases.

Primary outcome measures: For each patient, multimorbidity was measured either by the presence of $\geq 2$ or $\geq 3$ chronic health problems, from a list of 147 chronic health problems. Clinical data were collected using the general practitioner's knowledge of the patient's history, patient's self-report and medical records. Cluster analyses were performed to reveal distinct patterns of multimorbidity.

Secondary outcome measures: Patient social and demographic data (sex, age, residence area, current marital status, number of years of formal education, living arrangements, professional status and selfperceived economic status). Logistic regression analyses were performed to determine the association between sociodemographic factors and multimorbidity.

Results: Multimorbidity (2 or more chronic health problems) was present in $72.7 \%$. When a cut-off of three or more was used, an expressive percentage of multimorbidity $(57.2 \%)$ remained present. The likelihood of having multimorbidity increased significantly with age. Pensioners/retirees and adults with low levels of education were significantly more likely to suffer from multimorbidity. Cardiometabolic and mental disorders were the most common chronic health problems. Six multimorbidity clusters have been identified.

Conclusions: Multimorbidity was found to be a common occurrence in the Portuguese primary care users. Future primary healthcare policies should take multimorbidity into consideration.

\section{Strengths and limitations of this study}

- This is the first study to quantify multimorbidity in the adult population attending general practice in Portugal. It contributes to a better knowledge of the epidemiological factors associated with multimorbidity in Portugal.

- This study investigated a large list of 147 chronic health problems. Most multimorbidity studies only considered a small list of chronic conditions.

- Clinical data were collected using three data sources for each patient: general practitioner (GP)'s knowledge of patient's history, patient's self-report and medical records. Most multimorbidity studies rely on only one of these sources of data.

- The study findings may not be generalisable to the entirety of the Portuguese primary care, since a random sample of each GP's patients was not possible to achieve. The study lacks a disease severity score and the cross-sectional nature of the study design does not allow for causal inferences to be proven.

- The most common chronic health problems and the prevalence of multimorbidity encountered were similar to those found in other studies, which supports the validity of the study findings.

\section{BACKGROUND}

Primary care plays a central role in the management of chronic medical conditions. ${ }^{1} 2$ Evidence to date suggests that the majority of patients with chronic health conditions do not have a single diagnosis, but numerous diagnoses coexist within one person. ${ }^{3}$ Multimorbidity has been defined as the co-occurrence of more than one chronic condition in an individual, ${ }^{4}$ and this has become more common across studied population groups over time with important consequences for primary care and secondary care providers.

As the world population ages and life expectancy increases, multimorbidity becomes progressively common $^{5}$ in developed ${ }^{6-8}$ and 
developing countries. ${ }^{9-12}$ Nonetheless, multimorbidity can be present in all age groups, rendering it a widespread public health concern. ${ }^{13}$

The overall estimated prevalence of multimorbidity varies across the literature, depending on the studied population and the used methodology, ${ }^{14}$ and the primary care setting is no exception. In a recent Dutch study ${ }^{15}$ involving about 213000 patients, multimorbidity was found among $13 \%$ of the population, but it can rise to as high as $95 \%$ within the group of the oldest ones. ${ }^{16}$

Patients who suffer from multimorbidity have poor health outcomes, ${ }^{17}$ high healthcare utilisation ${ }^{18}$ (78\% of all primary care consultations are provided to patients with multiple chronic conditions ${ }^{6}$ ), more hospital admissions ${ }^{19}$ and increased average cost of care. ${ }^{20}$ This poses a significant challenge on patients' lives, general practitioners' (GPs) work and healthcare services in general. ${ }^{21}$

Since GPs are responsible for the complete person's health, they should pay special attention to the prevalence and patterns of multimorbidity in order to best plan and provide patient care.

Taking into consideration the demographic projections, the Portuguese population will significantly age over the course of the next four decades. By 2050, about $32 \%$ of the population is projected to be aged 65 and over, significantly above the Organisation for Economic Co-operation and Development (OECD) average of $25.7 \%,{ }^{22}$ which underlines the need for health practitioners to become acquainted with the multimorbidity phenomenon in Portugal.

This study aimed to determine the prevalence of multimorbidity in the adult population attending primary care in Portugal, to identify associated sociodemographic factors, and to reveal combinations of chronic health problems that in the future might benefit from directed care management.

\section{METHODS}

\section{Study design}

This cross-sectional study, conducted from October 2013 to December 2014, represents the first phase of the MM-PT project-Multimorbidity in primary care in PorTugal-designed to further the knowledge of the epidemiology of multimorbidity in the country from multiple perspectives. Details of study design, definitions and methods were previously published in this journal. ${ }^{23}$

The study was conducted in agreement with the principles of the Declaration of Helsinki ${ }^{24}$ and received institutional Ethics Committee approval. The reporting of this study conforms to the Strengthening the Reporting of Observational Studies in Epidemiology (STROBE) statement. ${ }^{25}$

\section{Participants}

The sampling approach is described in detail elsewhere. ${ }^{23}$ Briefly, in agreement with the distribution of the Portuguese population aged 18 years and older across the five mainland Portugal Healthcare Administrative Regions, a random sample of GPs, working in the National Health Service, was obtained aiming at a total of 1500 general practice patients recruited within them. GPs were initially invited by letter. Subsequently, frequent reminders were sent by letter, telephone, email and/or personal visits. In view of insufficient response to these recruitment strategies, changes in the study procedures were deemed necessary: recruitment awareness was raised both by emails sent to medical online discussion forums/mailing groups and via the distribution of leaflets in national conferences. No reimbursement of any kind was offered for involvement in this study.

Adult patients who attended primary care consultations during the study period and were judged as being physically and mentally able to provide informed consent were included in the study.

\section{Data collection procedures}

Data collection was performed by protocol. ${ }^{23}$ The questionnaire was pilot tested in 25 adult general practice patients. No alterations to the questionnaire were found to be needed.

\section{Outcome variable}

For each patient, multimorbidity was measured either by the presence of $\geq 2$ or $\geq 3$ chronic health problems at the time of data collection. The chronic health problems of interest were drawn from the 147 International Classification of Primary Care, Second edition (ICPC-2) rubrics list gathered by the Family Medicine Research Centre, University of Sydney. ${ }^{26}$

\section{Independent variables}

Social and demographic characteristics such as sex (male/female), age (18-34/35-49/50-64/ $\geq 65$ years), residence area (urban/rural), current marital status (married-cohabiting/single/widowed/separateddivorced), number of years of formal education (less than 6 years/at least 6 but not more than 9 years/more than 9 years), living arrangements (couple/extended family/alone/other), professional status (pensionerretired/employed/unemployed/housewife/student) and self-perceived economic status ('Just enough to make ends meet'/'Not enough to make ends meet'/ 'Some money left over') 'were assessed by the personal information section of the Portuguese version of the EASY-Care questionnaire.

The clinical data (chronic health problems) were collected using the GP's knowledge of the patient's history, patient's self-report and electronic and/or paper medical records of the patient. Health problems were defined as chronic by the O'Halloran criteria: (A) have a duration that has lasted, or is expected to last, at least 6 months; (B) have a pattern of recurrence or deterioration; (C) have a poor prognosis and (D) produce 
consequences or sequelae that impact on the individual's quality of life. ${ }^{26}$

\section{Statistical analyses}

In addition to the descriptive analysis, $\chi^{2}$ tests for group comparisons and logistic regressions, performed as specified in the aforementioned published study protocol, ${ }^{23}$ cluster analyses $^{27}$ were also executed aimed at revealing distinct patterns of chronic health problems. As previously employed by Marengoni et $a l^{28}$ a correlation matrix was computed among the most frequent chronic health problems in the sample (prevalence $>5 \%$ and $>10 \%$ ) using the Yule $Q$ measure of similarity and average linkage as an algorithm.

All analyses were done using the IBM SPSS Statistics for Windows, V.21.0 (IBM Corporation, Armonk, New York, USA).

$\mathrm{p}$ Values $<0.05$ were considered statistically significant.

\section{RESULTS}

\section{Characteristics of participants}

Enrolled GPs approached a total of 2027 patients aged 18 years and older for inclusion in the study; $98.3 \%$ (1279 women and 714 men) agreed to participate. Thirty-four refused to participate due to personal reasons. All five mainland Portuguese Healthcare Administrative Regions attained or surpassed the minimum required sample size: 559 in the North $(104 \%), 750$ in the Centre $(208 \%), 459$ in the Lisbon-Tejo Valley (109\%), 149 in Alentejo (124\%) and 76 in Algarve (127\%). The average participant-GP ratio was 28.9 patients per GP (range 10-65).

The mean age for the sample was 56.3 years (59.0 years for men and 54.8 years for women). Table 1 shows the demographic characteristics of sample respondents. The most frequent marital status was married or cohabiting in $69.5 \%$ of the sample. The most predominant living arrangement was living as a couple (57.2\%). A considerable proportion of the sample consisted of pensioners and retirees $(41.5 \%)$, and adults with a low level of education $(48.7 \%)$. Sufficient monthly income was reported in $54.4 \%$ of the cases.

\section{Prevalence of multimorbidity}

Almost 9 out of 10 study participants $(87.0 \%)$ had at least 1 chronic health problem, with an overall average of 3.4 (3.6 in men, 3.3 in women).

Multimorbidity, measured as a count of 2 or more chronic health problems, was present in $72.7 \%$ of the sample. Consistently, when a cut-off of 3 or more was used, an expressive percentage of multimorbidity $(57.2 \%)$ remained present.

There was a significant relationship $(\mathrm{p}<0.05)$ between each demographic characteristic and multimorbidity (table 2). Males, old age, residing in rural areas, becoming a widow or widower, living alone, having a low level of education, being a pensioner or a retiree, and
Table 1 Demographic characteristics of the sample

\begin{tabular}{lc}
\hline Characteristic & $\%(\mathbf{n})$ \\
\hline Sex & \\
Women & $64.2(1279)$ \\
$\quad$ Men & $35.8(714)$ \\
Age (years) & \\
18-34 & $14.5(288)$ \\
$35-49$ & $19.5(388)$ \\
$50-64$ & $30.7(612)$ \\
$\geq 65$ & $35.4(705)$ \\
Residence area & \\
Urban & $53.1(1058)$ \\
Rural & $46.9(935)$ \\
Marital status & \\
Married/cohabiting & $69.5(1385)$ \\
Single & $12.2(244)$ \\
Widowed & $11.6(232)$ \\
Separated/divorced & $6.6(132)$ \\
Living arrangements & \\
Couple & $57.2(1139)$ \\
Extended family & $24.1(481)$ \\
Alone & $14.5(289)$ \\
$\quad$ Other (including care home) & $4.2(84)$ \\
Education & \\
Low level (less than 6 years) & $48.7(971)$ \\
Medium level (at least 6 but not more than & $23.7(472)$ \\
9 years) & \\
High level (more than 9 years) & $27.6(550)$ \\
Professional status & \\
Pensioner/retired & \\
Employed (full-time/part-time) & \\
Unemployed & $31.5(828)$ \\
Housewife & $10.5(209)$ \\
Student & $6.1(121)$ \\
Monthly income & $2.6(51)$ \\
'Just enough to make ends meet' & \\
'Not enough to make ends meet' & $54.4(1084)$ \\
'Some money left over' & $27.5(549)$ \\
\hline & $18.1(360)$ \\
\hline
\end{tabular}

perceiving an insufficient monthly income were associated with higher rates of multimorbidity. A similar trend was observed for three or more chronic health problems multimorbidity cut-off, with the exception of an equally prevalent multimorbidity between monthly income levels $(\mathrm{p}=0.070)$.

As table 3 shows, the prevalence of multimorbidity (using 2 cut-off points) increased with age in men and women. Men aged 50-64 had a higher prevalence of multimorbidity compared to their female counterparts, whereas in all other age groups women surpassed men. However, none of these differences were statistically significant.

As can be seen from table 4, after adjustment, the demographic variables that remained associated with the presence of multimorbidity were age, education and professional status. The likelihood of having multimorbidity increased significantly with age $(p<0.001)$. Pensioners, retirees and adults with low levels of education were significantly more likely to suffer from multimorbidity. No 
Table 2 Prevalence of multimorbidity according to demographic characteristics

\begin{tabular}{|c|c|c|c|c|c|c|}
\hline \multirow[b]{2}{*}{ Characteristic } & \multirow{2}{*}{$\begin{array}{l}\text { Percentage of } \\
\text { healthy adults } \\
\text { (no chronic health } \\
\text { problems) }(95 \% \mathrm{Cl})\end{array}$} & \multicolumn{4}{|c|}{ Percentage of patients with multimorbidity $(95 \% \mathrm{Cl})$} & \multirow{2}{*}{$\begin{array}{l}\text { Mean number of } \\
\text { chronic health } \\
\text { problems }(95 \% \mathrm{Cl}) \\
\text { [median] }\end{array}$} \\
\hline & & $\begin{array}{l}\geq 2 \text { chronic health } \\
\text { problems }\end{array}$ & $\mathrm{p}$ Value & $\begin{array}{l}\geq 3 \text { chronic health } \\
\text { problems }\end{array}$ & p Value & \\
\hline Sex & & & 0.015 & & 0.003 & \\
\hline Women & $14.6(12.7$ to 16.6$)$ & 70.8 (68.3 to 73.3$)$ & & 54.7 (51.9 to 57.4$)$ & & 3.3 (3.1 to 3.4$)[3.0]$ \\
\hline Men & 10.1 (7.9 to 12.3$)$ & $75.9(72.8$ to 79.1$)$ & & 61.6 (58.0 to 65.2$)$ & & $3.6(3.4$ to 3.8$)[3.0]$ \\
\hline Age (years) & & & $<0.001$ & & $<0.001$ & \\
\hline $18-34$ & $47.6(41.8$ to 53.4$)$ & 24.7 (19.7 to 29.7$)$ & & $12.8(9.0$ to 16.7$)$ & & $1.0(0.9$ to 1.2$)[1.0]$ \\
\hline $35-49$ & $18.3(14.4$ to 22.2$)$ & 58.5 (53.6 to 63.4$)$ & & 32.0 (27.3 to 36.6$)$ & & $2.1(1.9$ to 2.3$)$ [2.0] \\
\hline $50-64$ & $6.4(4.4$ to 8.3$)$ & 81.2 (78.1 to 84.3$)$ & & 64.5 (60.7 to 68.3$)$ & & $3.6(3.4$ to 3.8$)[3.0]$ \\
\hline$\geq 65$ & $1.7(0.1$ to 2.7$)$ & 92.6 (90.7 to 94.6$)$ & & 82.7 (79.9 to 85.5$)$ & & 4.9 (4.7 to 5.1$)[5.0]$ \\
\hline Residence area & & & $<0.001$ & & $<0.001$ & \\
\hline Urban & $15.5(13.3$ to 17.7$)$ & $68.3(65.5$ to 71.1$)$ & & 51.3 (48.3 to 54.3 ) & & 3.1 (3.0 to 3.3$)[3.0]$ \\
\hline Rural & $10.2(8.2$ to 12.1$)$ & 77.5 (74.9 to 80.2$)$ & & 63.7 ( 60.7 to 66.8$)$ & & $3.7(3.5$ to 3.9$)[3.0]$ \\
\hline Marital status & & & $<0.001$ & & $<0.001$ & \\
\hline Married/cohabiting & $10.8(9.2$ to 12.5$)$ & 75.4 (73.1 to 77.7$)$ & & 58.7 (56.1 to 61.3$)$ & & 3.4 (3.3 to 3.6$)[3.0]$ \\
\hline Single & 34.4 (28.4 to 40.4$)$ & 41.0 (34.8 to 47.2$)$ & & 28.3 (22.6 to 34.0$)$ & & 1.9 (1.6 to 2.2$)[1.0]$ \\
\hline Widowed & $3.0(0.8$ to 5.2$)$ & $92.2(88.8$ to 95.7$)$ & & 81.9 (76.9 to 86.9$)$ & & $5.0(4.6$ to 5.3$)[5.0]$ \\
\hline Separated/divorced & 13.6 (7.7 to 19.6$)$ & 68.2 (60.1 to 76.2$)$ & & 50.8 (42.1 to 59.4$)$ & & $3.0(2.6$ to 3.4$)[3.0]$ \\
\hline Living arrangements & & & $<0.001$ & & $<0.001$ & \\
\hline Couple & $11.8(9.9$ to 13.6$)$ & 75.2 (72.7 to 77.8$)$ & & 59.0 (56.1 to 61.9$)$ & & 3.4 (3.3 to 3.6$)[3.0]$ \\
\hline Extended family & 17.3 (13.9 to 20.6$)$ & 62.6 (58.2 to 66.9$)$ & & 48.2 (43.8 to 52.7$)$ & & $3.0(2.7$ to 3.2$)[2.0]$ \\
\hline Alone & $10.0(6.5$ to 13.5$)$ & 80.3 (75.7 to 84.9$)$ & & 66.4 (61.0 to 71.9$)$ & & 4.0 (3.7 to 4.4$)[4.0]$ \\
\hline Other (including care home) & 15.5 (7.6 to 23.4$)$ & $69.0(59.0$ to 79.1$)$ & & $51.2(40.3$ to 62.1$)$ & & $2.9(2.4$ to 3.4$)$ [3.0] \\
\hline Education & & & $<0.001$ & & $<0.001$ & \\
\hline Low level (less than 6 years) & $3.9(2.7$ to 5.1$)$ & 89.3 (87.3 to 91.2$)$ & & 75.9 (73.2 to 78.6$)$ & & 4.5 (4.3 to 4.6$)[4.0]$ \\
\hline Medium level (at least 6 but not more than 9 years) & $14.6(11.4$ to 17.8$)$ & 65.9 (61.6 to 70.2$)$ & & 48.7 (44.2 to 53.3$)$ & & $2.7(2.5$ to 2.9$)[2.0]$ \\
\hline High level (more than 9 years) & 27.6 (23.9 to 31.4$)$ & 49.1 (44.9 to 53.3 ) & & 31.3 (27.4 to 35.2$)$ & & $2.1(1.9$ to 2.2$)[1.0]$ \\
\hline Professional status & & & $<0.001$ & & $<0.001$ & \\
\hline Pensioner/retired & $1.9(1.0$ to 2.9$)$ & $92.0(90.2$ to 93.9$)$ & & 81.2 (78.5 to 83.8 ) & & $4.8(4.6$ to 5.0$)[5.0]$ \\
\hline Employed (full-time/part-time) & 20.5 (17.7 to 23.4$)$ & 56.8 (53.3 to 60.2$)$ & & 37.9 (34.5 to 41.3 ) & & 2.3 (2.1 to 2.4$)[2.0]$ \\
\hline Unemployed & 19.6 (14.2 to 25.0$)$ & 65.1 (58.6 to 71.6$)$ & & 45.9 (39.1 to 52.7 ) & & 2.7 (2.4 to 3.0$)$ [2.0] \\
\hline Housewife & $8.3(3.3$ to 13.2$)$ & $81.0(73.9$ to 88.1$)$ & & 60.3 (51.5 to 69.2$)$ & & 3.4 (3.0 to 3.9 ) [3.0] \\
\hline Student & 60.8 (46.9 to 74.7$)$ & $13.7(4.0$ to 23.5$)$ & & $2.0(0.0$ to 5.9$)$ & & $0.5(0.3$ to 0.8$)[0.0]$ \\
\hline Monthly income & & & 0.018 & & 0.070 & \\
\hline 'Just enough to make ends meet' & $12.8(10.8$ to 14.8$)$ & $72.4(69.8$ to 75.1$)$ & & 57.4 (54.4 to 60.3$)$ & & 3.4 (3.3 to 3.6$)[3.0]$ \\
\hline 'Not enough to make ends meet' & $11.7(9.0$ to 14.4$)$ & 76.3 (72.8 to 79.9$)$ & & 59.9 (55.8 to 64.0$)$ & & 3.5 (3.3 to 3.7 ) [3.0] \\
\hline 'Some money left over' & 15.6 (11.8 to 19.3$)$ & 67.8 (62.9 to 72.6$)$ & & $52.2(47.0$ to 57.4$)$ & & 3.2 (2.9 to 3.4$)$ [3.0] \\
\hline
\end{tabular}


differences in the odds of multimorbidity were detected in terms of sex, residence area, marital status, living arrangements or monthly income.

Chronic health problems and patterns of multimorbidity

Table 5 shows the most common chronic health problems in the sample, considering a minimum prevalence of at least $5 \%$. Globally, the two leading problems were lipid disorder (44.8\% males, $35.7 \%$ females) and uncomplicated hypertension (43.8\% males, $33.4 \%$ females). Depressive disorder was the third most common chronic health problem among females $(22.8 \%)$ and non-insulin dependent diabetes among males (25.4\%). In both sexes, obesity came in fourth place (19.5\% males, $14.4 \%$ females). The least common problem was osteoporosis with a prevalence of around $5 \%$.

As could be expected, each common chronic health problem co-occurred with some other chronic health problem at a greater extent than as a stand-alone condition (table 5).

Women with a diagnosis of non-insulin dependent diabetes, osteoarthritis of the knee, back syndrome with radiating pain and lipid disorder were 74.4 times, 62.3 times, 42.5 times and 21.1 times, respectively, more likely to have multimorbidity ( 2 or more chronic health problems) than women without these diagnoses $(p<0.001)$. When considering multimorbidity as the co-occurrence of three or more chronic health problems, there was a decrease in the odds. Nonetheless, women with osteoarthritis of the knee, lipid disorder, non-insulin dependent diabetes and back syndrome with radiating pain were 13.4 times, 11.3 times, 9.9 times and 8.7 times, respectively, more likely to have three or more chronic health problems than women without these diagnoses $(\mathrm{p}<0.001)$ (table 6).

In men, the highest ORs for multimorbidity (2 or more chronic health problems) were associated with obesity (OR, 28.7 (95\% CI 7.0 to 117.5); $\mathrm{p}<0.001$ ), lipid disorder (OR, 16.0 (95\% CI 8.9 to 28.8); $\mathrm{p}<0.001)$ and benign prostatic hypertrophy (OR, 14.8 (95\% CI 4.7 to 47.3); $\mathrm{p}<0.001)$. Men with complicated hypertension were 26.2 times more likely to have three or more chronic health problems than non-hypertensive men (table 6).

Figure 1 shows the six disease clusters that were identified using cluster analysis. One cluster was comprised solely of overweight. Two clusters included two diseases: one anxiety disorder/anxiety state and depressive disorder, and one varicose veins of the leg and back syndrome without radiating pain. One cluster included three diseases: obesity, non-insulin dependent diabetes and uncomplicated hypertension. Two clusters included four diseases: one osteoarthrosis (other), osteoporosis, goitre and back syndrome with radiating pain and one complicated hypertension, lipid disorder, osteoarthritis of the knee and benign prostatic hypertrophy.

As a corroboration procedure, a second cluster analysis was performed using chronic health problems with a prevalence $>10 \%$. Obesity, non-insulin dependent 
Table 4 Logistic regression model for demographic determinants of multimorbidity

\begin{tabular}{|c|c|c|c|c|c|c|}
\hline \multirow[b]{3}{*}{ Characteristic } & \multicolumn{6}{|c|}{ Multimorbidity } \\
\hline & \multicolumn{3}{|c|}{$\geq 2$ chronic health problems } & \multicolumn{3}{|c|}{$\geq 3$ chronic health problems } \\
\hline & $\overline{\text { OR }}$ & $95 \% \mathrm{Cl}$ & p Value & $\overline{O R}$ & $95 \% \mathrm{Cl}$ & p Value \\
\hline \multicolumn{7}{|l|}{ Sex } \\
\hline Women & base & - & - & base & - & - \\
\hline Men & 0.9 & 0.7 to 1.2 & 0.649 & 1.0 & 0.8 to 1.3 & 0.990 \\
\hline \multicolumn{7}{|l|}{ Age (years) } \\
\hline $18-34$ & base & - & - & base & - & - \\
\hline $35-49$ & 3.3 & 2.2 to 4.8 & $<0.001$ & 2.5 & 1.6 to 3.8 & $<0.001$ \\
\hline $50-64$ & 6.7 & 4.4 to 10.1 & $<0.001$ & 7.0 & 4.5 to 11.0 & $<0.001$ \\
\hline$\geq 65$ & 9.3 & 5.0 to 17.4 & $<0.001$ & 11.1 & 6.3 to 19.7 & $<0.001$ \\
\hline \multicolumn{7}{|l|}{ Residence area } \\
\hline Urban & base & - & - & base & - & - \\
\hline \multirow{2}{*}{\multicolumn{7}{|c|}{ Marital status }} \\
\hline & & & & & & \\
\hline Married/cohabiting & 1.4 & 0.9 to 2.3 & 0.182 & 1.0 & 0.6 to 1.7 & 0.958 \\
\hline Single & base & - & - & base & - & - \\
\hline Widowed & 1.3 & 0.7 to 2.6 & 0.410 & 1.2 & 0.7 to 2.1 & 0.521 \\
\hline Separated/divorced & 1.0 & 0.6 to 1.7 & 0.985 & 1.0 & 0.6 to 1.8 & 0.948 \\
\hline \multicolumn{7}{|l|}{ Living arrangements } \\
\hline Couple & 1.4 & 0.9 to 2.3 & 0.182 & 0.9 & 0.6 to 1.5 & 0.778 \\
\hline Extended family & 1.0 & 0.6 to 1.7 & 0.985 & 1.0 & 0.6 to 1.6 & 0.979 \\
\hline Alone & base & - & - & base & - & - \\
\hline Other (including care home) & 1.3 & 0.7 to 2.6 & 0.410 & 0.9 & 0.5 to 1.7 & 0.819 \\
\hline \multicolumn{7}{|l|}{ Education } \\
\hline Low level (less than 6 years) & 2.0 & 1.4 to 2.8 & $<0.001$ & 1.7 & 1.3 to 2.4 & 0.001 \\
\hline Medium level (at least 6 but not more than 9 years) & 1.3 & 1.0 to 1.8 & 0.081 & 1.4 & 1.1 to 2.0 & 0.014 \\
\hline High level (more than 9 years) & base & - & - & base & - & - \\
\hline \multicolumn{7}{|l|}{ Professional status } \\
\hline Pensioner/retired & 4.4 & 1.6 to 11.9 & 0.003 & 15.2 & 2.0 to 117.6 & 0.009 \\
\hline Employed (full-time/part-time) & 1.9 & 0.8 to 4.7 & 0.143 & 8.2 & 1.01 to $62-2$ & 0.041 \\
\hline Unemployed & 2.5 & 1.0 to 6.2 & 0.053 & 10.8 & $1.4-82.3$ & 0.022 \\
\hline Housewife & 2.5 & $0.9-7.1$ & 0.073 & 8.5 & 1.1 to 66.8 & 0.043 \\
\hline Student & base & - & - & base & - & - \\
\hline \multicolumn{7}{|l|}{ Monthly income } \\
\hline 'Just enough to make ends meet' & 0.8 & 0.6 to 1.1 & 0.158 & - & - & - \\
\hline 'Not enough to make ends meet' & base & - & - & - & - & - \\
\hline 'Some money left over' & 0.8 & 0.5 to 1.1 & 0.182 & - & - & - \\
\hline
\end{tabular}

diabetes and uncomplicated hypertension remained together in a cluster and so did complicated hypertension and lipid disorder. Overweight, back syndrome with radiating pain and depressive disorder remained in independent clusters.

\section{DISCUSSION}

Strengths of the study

This is the first study to report the prevalence and patterns of multimorbidity in the Portuguese population aged 18 and older attending primary care consultations. It is cross-sectional, which is the most frequent design to assess the epidemiology of multimorbidity. ${ }^{29}$ A simple count of individual chronic conditions was the approach used to measure multimorbidity, which is also the most common across the literature. ${ }^{30}$

Although there is no agreement regarding the number and type of chronic health problems that should be included in multimorbidity related studies, the use of 12 or more frequent chronic diseases has been suggested by some authors ${ }^{31}$ as being the cut-off for better prevalence estimates; this study largely meets this principle, since a list of 147 chronic health problems was used.

\section{Statement of overall findings}

In general, the study results substantiate the commonly assumed perception that multimorbidity is the rule in primary care. ${ }^{3}$ Even though it may not be entirely accurate to directly compare the prevalence estimates of multimorbidity between studies, owing to the differences in the employed methodologies, ${ }^{14}$ some authors have stated that prevalence estimates are similar when multimorbidity is defined as two or more disease entities, independently of how a disease entity is defined. ${ }^{32}$ In this study, the high prevalence of two or more chronic 
Table 5 Chronic health problems with a prevalence $>5 \%$ and their co-occurrence rates

\begin{tabular}{|c|c|c|c|c|c|c|c|}
\hline \multirow[b]{2}{*}{ ICPC2 code } & \multirow[b]{2}{*}{ Chronic health problem } & \multirow[b]{2}{*}{$\mathbf{N}$} & \multirow[b]{2}{*}{ Stand-alone (\%) } & \multicolumn{4}{|c|}{$\begin{array}{l}\text { Associated chronic health } \\
\text { problems (\%) }\end{array}$} \\
\hline & & & & +1 & +2 & +3 & +4 or more \\
\hline T93 & Lipid disorder & 776 & 3.7 & 9.8 & 14.7 & 18.9 & 52.8 \\
\hline K86 & Hypertension, uncomplicated & 740 & 6.4 & 14.1 & 16.8 & 19.1 & 43.8 \\
\hline P76 & Depressive disorder & 340 & 10.9 & 12.9 & 13.8 & 18.5 & 43.8 \\
\hline T90 & Diabetes, non-insulin dependent & 333 & 2.7 & 8.4 & 13.2 & 19.2 & 56.5 \\
\hline T82 & Obesity & 323 & 4.3 & 9.6 & 16.4 & 18.6 & 51.1 \\
\hline L86 & Back syndrome with radiating pain & 250 & 3.6 & 10.0 & 10.8 & 14.0 & 61.6 \\
\hline T83 & Overweight & 213 & 4.7 & 11.3 & 16.0 & 18.3 & 49.8 \\
\hline K87 & Hypertension, complicated & 206 & 0 & 2.9 & 8.7 & 16.0 & 72.3 \\
\hline K95 & Varicose veins of leg & 195 & 4.1 & 9.7 & 14.9 & 17.9 & 53.3 \\
\hline L90 & Osteoarthritis of knee & 191 & 0.5 & 6.3 & 6.3 & 11.0 & 75.9 \\
\hline P74 & Anxiety disorder/anxiety state & 176 & 6.8 & 13.1 & 13.1 & 17.0 & 50.0 \\
\hline L91 & Osteoarthrosis, other & 130 & 1.5 & 3.8 & 10.0 & 15.4 & 69.2 \\
\hline Y85 & Benign prostatic hypertrophy & 116 & 2.6 & 6.9 & 9.5 & 18.1 & 62.9 \\
\hline L84 & Back syndrome without radiating pain & 108 & 5.6 & 7.4 & 10.2 & 24.1 & 52.8 \\
\hline T81 & Goitre & 107 & 1.9 & 15.0 & 15.0 & 13.1 & 55.1 \\
\hline L95 & Osteoporosis & 105 & 1.9 & 4.8 & 7.6 & 16.2 & 69.5 \\
\hline
\end{tabular}

health problems-multimorbidity-is consistent with data present in the literature. ${ }^{29}$

Given the current absence of a consensual definition of multimorbidity, the recommendation of Fortin $e t a l^{31}$ of using two operational definitions of multimorbidity was followed. Therefore, in this study, multimorbidity was also defined by three or more chronic health problems. Although only a few studies have used this definition, ${ }^{29}$ it is of relevance for practitioners since it can identify patients with higher needs. ${ }^{31}$ More than half of the study participants had three or more chronic health problems, which is a common prevalence found in developed countries. ${ }^{3} 33$

These findings suggest that Portuguese GPs are likely to struggle with increasingly daily practice concerns, since they will face many challenges regarding the

Table 6 ORs (and 95\% Cls) for multimorbidity associated with 10 most common chronic health problems in women and men

\begin{tabular}{|c|c|c|c|c|c|c|c|c|c|}
\hline \multirow[b]{3}{*}{ ICPC2 code } & \multirow[b]{3}{*}{ Chronic health problem } & \multicolumn{8}{|c|}{ Multimorbidity } \\
\hline & & \multicolumn{4}{|c|}{$\geq 2$ chronic health problems } & \multicolumn{4}{|c|}{$\geq 3$ chronic health problems } \\
\hline & & $\%$ & OR & $95 \% \mathrm{Cl}$ & $p$ value & $\%$ & OR & $95 \% \mathrm{Cl}$ & p value \\
\hline T93 & Lipid disorder & 96.5 & 21.1 & 12.6 to 35.3 & $<0.001$ & 86.8 & 11.3 & 8.3 to 15.4 & $<0.001$ \\
\hline K86 & Hypertension, uncomplicated & 93.7 & 10.1 & 6.7 to 15.3 & $<0.001$ & 78.7 & 5.0 & 3.8 to 6.5 & $<0.001$ \\
\hline P76 & Depressive disorder & 88.4 & 4.0 & 2.7 to 5.8 & $<0.001$ & 75.0 & 3.2 & 2.4 to 4.2 & $<0.001$ \\
\hline K95 & Varicose veins of leg & 96.3 & 12.7 & 5.6 to 29.0 & $<0.001$ & 85.2 & 5.7 & 3.6 to 8.9 & $<0.001$ \\
\hline T90 & Diabetes, non-insulin dependent & 99.3 & 74.4 & 10.4 to 533.7 & $<0.001$ & 90.8 & 9.9 & 5.7 to 17.4 & $<0.001$ \\
\hline P74 & Anxiety disorder/anxiety state & 91.9 & 5.3 & 2.8 to 9.9 & $<0.001$ & 76.5 & 3.0 & 2.0 to 4.5 & $<0.001$ \\
\hline L90 & Osteoarthritis of knee & 99.2 & 62.3 & 8.7 to 447.5 & $<0.001$ & 93.1 & 13.4 & 6.8 to 26.7 & $<0.001$ \\
\hline T83 & Overweight & 95.2 & 9.3 & 4.1 to 21.4 & $<0.001$ & 81.7 & 4.2 & 2.6 to 6.7 & $<0.001$ \\
\hline T82 & Obesity & 98.6 & 28.7 & 7.0 to 117.5 & $<0.001$ & 91.4 & 8.9 & 4.8 to 16.4 & $<0.001$ \\
\hline Y85 & Benign prostatic hypertrophy & 97.4 & 14.8 & 4.7 to 47.3 & $<0.001$ & 90.5 & 7.5 & 3.9 to 14.2 & $<0.001$ \\
\hline K87 & Hypertension, complicated & 100.0 & - & - & - & 97.1 & 26.2 & 8.2 to 83.6 & $<0.001$ \\
\hline T83 & Overweight & 95.4 & 7.6 & 2.7 to 21.0 & $<0.001$ & 87.4 & 5.0 & 2.6 to 9.6 & $<0.001$ \\
\hline L86 & Back syndrome with radiating pain & 91.1 & 3.6 & 1.6 to 8.0 & 0.001 & 79.7 & 2.7 & 1.5 to 4.8 & $<0.001$ \\
\hline L90 & Osteoarthritis of knee & 100.0 & - & - & - & 93.3 & 9.8 & 3.5 to 27.5 & $<0.001$ \\
\hline P76 & Depressive disorder & 93.8 & 5.1 & 1.6 to 16.6 & 0.003 & 83.3 & 3.3 & 1.5 to 7.2 & 0.001 \\
\hline
\end{tabular}




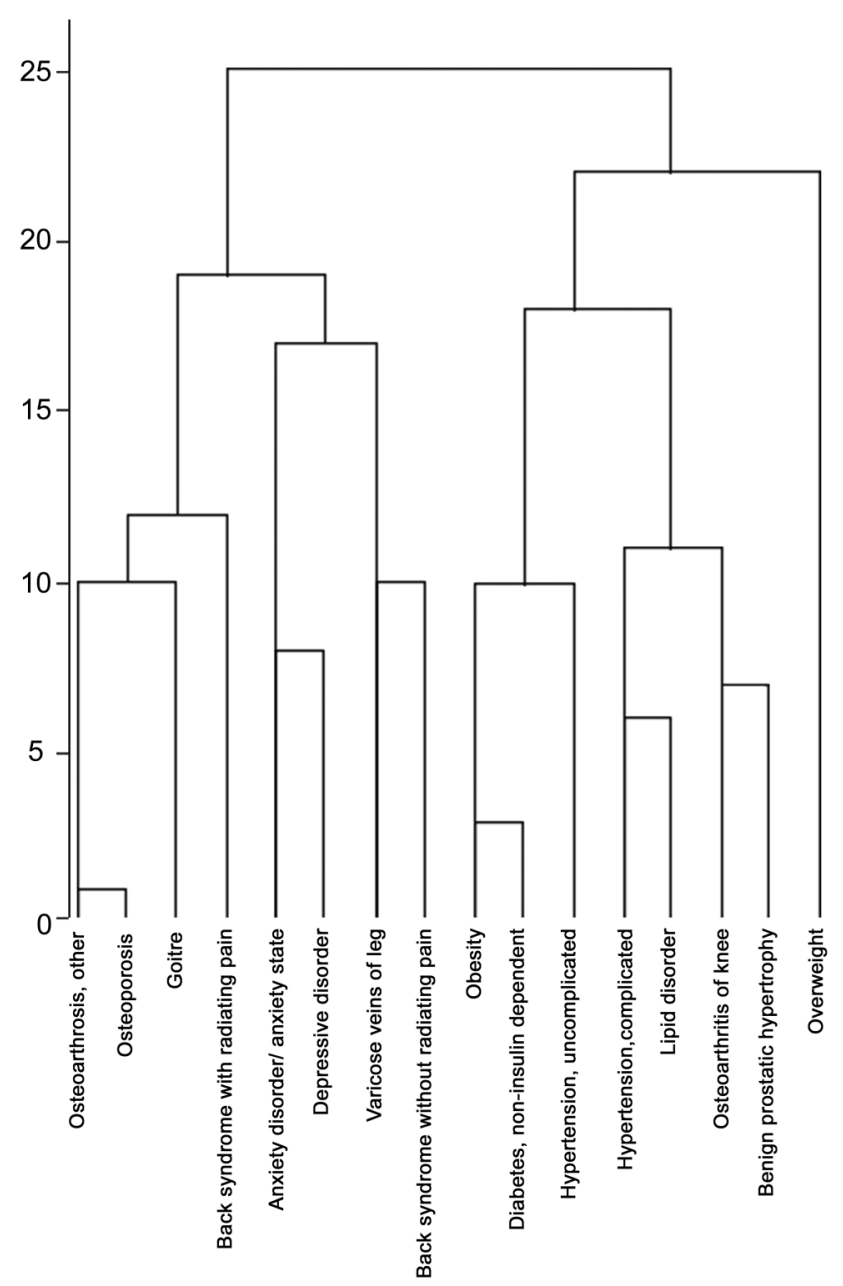

Figure 1 Dendogram resulting from cluster analysis.

multimorbidity impact on disease management, as is the case in other countries. ${ }^{34}$ Caring for patients with multimorbidity is a demanding task because, on the one hand, there is a lack of multimorbidity related guidelines $^{35}$ and, on the other, current interventions have had mixed effectiveness results. ${ }^{36}$ There remains the need to identify patients with multimorbidity in order to develop new and stronger patient-oriented interventions. ${ }^{36}$ These can be supported by a longitudinal doctor-patient relationship-based care, the offer of a benefit-risk balance to each individual, and decision taking driven by the patient's capability to cope with them, ultimately avoiding overdiagnosis and overtreatment. ${ }^{37}$ Recent efforts to address multimorbidity within guidelines are also being pursued. ${ }^{38}$

\section{Relationship with other studies}

In line with previous reports, ${ }^{3} 7131518$ 39-42 a significant association between age and prevalence of multimorbidity, irrespective of the definitions used, was found in this study. The presence of multiple chronic health problems increased with age and was highest among adults aged 65 or older. This is due to the accumulation of chronic health conditions during the ageing process. ${ }^{43}$ The prevalence of multimorbidity in each individual age group match those observed in a prior study, ${ }^{3}$ but are higher than the ones obtained by the majority of the published studies. ${ }^{29}$ Whether this is a real difference between the adult Portuguese primary care users and the primary care practice settings from other western countries, or the consequence of different methodologies of estimating multimorbidity prevalence, will remain the object of further research.

Women are associated with increased risk for multimorbidity. ${ }^{41}$ The majority of the study participants were female. Nonetheless, males were associated with higher rates of multimorbidity. This gender effect, however, did not persist after adjusting for all sociodemographic variables. These results therefore need to be interpreted with caution.

Krieger et $a t^{44}$ listed the level of education, occupational prestige and income as indicators of socioeconomic status. Lower socioeconomic status is a known determinant of multimorbidity, ${ }^{29}$ and this study's findings support it. Therefore, the level of education ${ }^{45}$ and professional status of the patients with multimorbidity should be taken into consideration when developing prevention strategies in primary care practice settings.

The type of source of data collection interferes with the prevalence estimates of multimorbidity. ${ }^{42}$ To minimise this effect, three sources of data were simultaneously used for each patient in this study: self-reported health status, analyses of medical records and GP's knowledge of the patient's history.

In agreement with previous reports, ${ }^{46}$ cardiometabolic and mental disorders were the most common chronic health problems in the study sample. Compared with national estimates, lipid disorder is lower in the sample $\left(38.9 \%\right.$ vs $\left.47.0 \%{ }^{47}\right)$, whereas hypertension (uncomplicated plus complicated) $-47.5 \%$ vs $42.2 \%,{ }^{48}$ depressive disorder-17.1\% vs $7.9 \%,{ }^{49}$ diabetes (non-insulin dependent) $-16.7 \%$ vs $11.7 \%^{50}$ and obesity $-16.2 \%$ vs $14.2 \%^{51}$ are higher in the sample. However, these studies present considerable differences in methodologies and target populations, which make prevalence comparisons difficult and the focus of further research. A possible reason for the relative high prevalence of depressive disorder in the sample may be the worsening of the socioeconomic conditions that Portugal faced due to the austerity programme from the International Monetary Fund and European Union. ${ }^{52}$ Another possible explanation for this is that depressive disorder is most frequently present in females, ${ }^{53}$ who constitute the majority of the sample.

The most common chronic health problems observed in the sample systematically coexisted with other chronic conditions in line with previous studies. ${ }^{46}$ Both females and males affected by one of the most common chronic health problems have an increased risk of having multimorbidity (two or more and three or more chronic conditions).

According to a recent review, ${ }^{29}$ there is no consistent pattern of multimorbidity across studies, mostly due to 
differences in the study design and inclusion criteria. ${ }^{54}$ Nevertheless, comorbidities can be grouped into three broad types: cardiometabolic, anxiety-depression and pain related. ${ }^{29}$ The results of this study further support this classification in adult patients with multimorbidity in the primary care. The cardiometabolic pattern found reflects three of the main features of the metabolic syndrome: ${ }^{54}$ diabetes, obesity and hypertension. This clustering of illness and risk factors is considered to be the agent of a new epidemic of cardiovascular disease.$^{55}$ The anxiety-depression cluster favours the diagnosis of a syndromal combined anxiety and depressive disorder, very common in primary care and seen most frequently in this setting. ${ }^{56}$ A cluster with pain was also present in this study; back syndrome with radiating pain was found to be associated with other chronic health problems that can cause or contribute to it such as osteoarthrosis and osteoporosis (indirectly by leading to painful fractures of the vertebrae). These findings strengthen the idea of the presence of associations of chronic health problems in patients with multimorbidity.

\section{Limitations of the study}

Some limitations of the study need to be stated. First, volunteer GPs collected data only from patients whom they have observed in consultations, which may have caused an over-representation of the frequent users of primary care services. The use of a random sample of each GP's patients might have produced more accurate results, ${ }^{3}$ although this would have not been practicable due to financial and time restraints. Nonetheless, the results of this study produce an image of the type of health problems tackled in GP practices. Second, even though all five mainland Portuguese Healthcare Administrative Regions were represented in this study, local unknown biases could have been introduced and our findings may not be generalisable to all Portuguese primary care. Third, although important when studying multimorbidity, no indicator of disease severity was used as it would be too demanding for participating GPs to evaluate in each consultation. Finally, this study is crosssectional, and therefore no causal relationship could be proven. Despite the stated limitations, the study suggests that the most common chronic health problems and the encountered prevalence of multimorbidity were similar to those found in other studies, which supports the validity of the study findings.

\section{CONCLUSION}

This study contributes to an increase in the understanding of multimorbidity and chronic health problems of the Portuguese population aged 18 and older attending primary care consultations. Multimorbidity was found to be a common occurrence in the studied sample. The highest levels of multimorbidity were identified among some vulnerable groups: the elderly, the less educated and the pensioners/retirees, which make them the target audience to consider in terms of public policies in the fields of health promotion and disease prevention. Regarding the prevalence of cardiometabolic and mental disorders, they clearly are two areas that need to be prioritised by GPs in the community. Six multimorbidity clusters have been identified. In the future, these patterns may benefit from directed care management, but only further research with a longitudinal approach will tell.

Survivorship with multimorbidity is the price of success of effective primary and secondary care, but do primary care patients with multimorbidity have a positive quality of life in Portugal? This is a question that phase II of our study will aim to answer.

Acknowledgements The authors acknowledge all the general practitioners who participated in the study, as well as their patients.

Contributors FP and LS led the design of the study. FP performed the statistical analyses and drafted the manuscript. LS revised the manuscript critically for important intellectual content. All authors read and approved the final manuscript draft.

Funding This research received no specific grant from any funding agency in the public, commercial or not-for-profit sectors.

\section{Competing interests None declared.}

Ethics approval The study was conducted in agreement with the principles of the Declaration of Helsinki. It was approved by the Ethics Committee of the Faculty of Health Sciences, University of Beira Interior and the Ethics Committee of the Central Health Region of Portugal.

Provenance and peer review Not commissioned; externally peer reviewed.

Data sharing statement No additional data are available.

Open Access This is an Open Access article distributed in accordance with the Creative Commons Attribution Non Commercial (CC BY-NC 4.0) license, which permits others to distribute, remix, adapt, build upon this work noncommercially, and license their derivative works on different terms, provided the original work is properly cited and the use is non-commercial. See: http:// creativecommons.org/licenses/by-nc/4.0/

\section{REFERENCES}

1. Rothman AA, Wagner EH. Chronic illness management: what is the role of primary care? Ann Intern Med 2003;138:256-61.

2. Demaio AR, Nielsen KK, Tersbol BP, et al. Primary Health Care: a strategic framework for the prevention and control of chronic non-communicable disease. Glob Health Action 2014;7:24504.

3. Fortin M, Bravo G, Hudon C, et al. Prevalence of multimorbidity among adults seen in family practice. Ann Fam Med 2005;3:223-8.

4. Mercer SW, Smith SM, Wyke S, et al. Multimorbidity in primary care: developing the research agenda. Fam Pract 2009;26:79-80.

5. Marengoni A, Angleman S, Melis R, et al. Aging with multimorbidity: a systematic review of the literature. Ageing Res Rev 2011;10:430-9.

6. Salisbury C, Johnson L, Purdy S, et al. Epidemiology and impact of multimorbidity in primary care: a retrospective cohort study. $\mathrm{Br} J$ Gen Pract 2011;61:e12-21.

7. Barnett K, Mercer SW, Norbury M, et al. Epidemiology of multimorbidity and implications for health care, research, and medical education: a cross-sectional study. Lancet 2012;380:37-43.

8. Salive ME. Multimorbidity in Older Adults. Epidemiol Rev 2013;35:75-83.

9. Khanam MA, Streatfield PK, Kabir ZN, et al. Prevalence and patterns of multimorbidity among elderly people in rural Bangladesh: a cross-sectional study. J Health Popul Nutr 2011;29:406-14.

10. Jerliu N, Toci E, Burazeri G, et al. Prevalence and socioeconomic correlates of chronic morbidity among elderly people in Kosovo: a population-based survey. BMC Geriatr 2013;13:22.

11. de S Santos Machado V, Valadares AL, Costa-Paiva LH, et al. Aging, obesity, and multimorbidity in women 50 years or older: a population-based study. Menopause 2013;20:818-24. 
12. Arokiasamy $P$, Uttamacharya $P$, Jain K. Multi-morbidity, functional limitations, and self-rated health among older adults in India: cross-sectional analysis of LASI pilot survey, 2010. SAGE Open 2015;5.

13. Prados-Torres A, Poblador-Plou B, Calderon-Larranaga A, et al. Multimorbidity patterns in primary care: interactions among chronic diseases using factor analysis. PLOS ONE 2012;7:e32190.

14. Stewart M, Fortin M, Britt HC, et al. Comparisons of multi-morbidity in family practice-issues and biases. Fam Pract 2013;30:473-80.

15. van Oostrom SH, Picavet HS, van Gelder BM, et al. Multimorbidity and comorbidity in the Dutch population-data from general practices. BMC Public Health 2012;12:715.

16. Formiga F, Ferrer A, Sanz H, et al. Patterns of comorbidity and multimorbidity in the oldest old: the Octabaix study. Eur J Intern Med 2013;24:40-4.

17. Fortin $\mathrm{M}$, Soubhi $\mathrm{H}$, Hudon $\mathrm{C}$, et al. Multimorbidity's many challenges. BMJ 2007;334:1016-17.

18. Glynn LG, Valderas JM, Healy $P$, et al. The prevalence of multimorbidity in primary care and its effect on health care utilization and cost. Fam Pract 2011;28:516-23.

19. Wang $\mathrm{HH}$, Wang JJ, Lawson KD, et al. Relationships of multimorbidity and income with hospital admissions in 3 health care systems. Ann Fam Med 2015;13:164-7.

20. Steiner CA, Friedman B. Hospital utilization, costs, and mortality for adults with multiple chronic conditions, Nationwide Inpatient Sample, 2009. Prev Chronic Dis 2013;10:E62.

21. Shiner A, Ford J, Steel N, et al. Managing multimorbidity in primary care. InnovAiT 2014;7:691-700.

22. OECD/EU. A Good Life in Old Age?: monitoring and improving quality in long-term care. Paris: OECD Health Policy Studies, OECD Publishing, 2013.

23. Prazeres F, Santiago L. Multimorbidity in primary care in Portugal (MM-PT): a cross-sectional three-phase observational study protocol. BMJ Open 2014;4:e004113.

24. World Medical Association. World Medical Association Declaration of Helsinki: ethical principles for medical research involving human subjects. JAMA 2013;310:2191-4.

25. von Elm E, Altman DG, Egger M, et al. The Strengthening the Reporting of Observational Studies in Epidemiology (STROBE) statement: guidelines for reporting observational studies. J Clin Epidemiol 2008;61:344-9.

26. O'Halloran J, Miller GC, Britt H. Defining chronic conditions for primary care with ICPC-2. Fam Pract 2004;21:381-6.

27. Cornell JE, Pugh JA, Williams JW, et al. Multimorbidity clusters: clustering binary data from multimorbidity clusters: clustering binary data from a large administrative medical database. Appl Multivariate Res 2007;12:163-82.

28. Marengoni A, Nobili A, Pirali C, et al. Comparison of disease clusters in two elderly populations hospitalized in 2008 and 2010. Gerontology 2013;59:307-15.

29. Violan C, Foguet-Boreu Q, Flores-Mateo G, et al. Prevalence, determinants and patterns of multimorbidity in primary care: a systematic review of observational studies. PLOS ONE 2014;9: e102149.

30. Huntley AL, Johnson R, Purdy S, et al. Measures of multimorbidity and morbidity burden for use in primary care and community settings: a systematic review and guide. Ann Fam Med 2012;10:134-41.

31. Fortin M, Stewart M, Poitras ME, et al. A systematic review of prevalence studies on multimorbidity: toward a more uniform methodology. Ann Fam Med 2012;10:142-51.

32. Harrison $\mathrm{C}$, Britt $\mathrm{H}$, Miller $\mathrm{G}$, et al. Examining different measures of multimorbidity, using a large prospective cross-sectional study in Australian general practice. BMJ Open 2014;4:e004694.

33. Boyd CM, McNabney MK, Brandt N, et al. Guiding principles for the care of older adults with multimorbidity: an approach for clinicians: American Geriatrics Society Expert Panel on the Care of Older Adults with Multimorbidity. J Am Geriatr Soc 2012;60:E1-E25.
34. Ornstein SM, Nietert PJ, Jenkins RG, et al. The prevalence of chronic diseases and multimorbidity in primary care practice: a PPRNet report. J Am Board Fam Med 2013;26:518-24.

35. Lugtenberg M, Burgers JS, Clancy $\mathrm{C}$, et al. Current guidelines have limited applicability to patients with comorbid conditions: a systematic analysis of evidence-based guidelines. PLOS ONE 2011;6:e25987.

36. Smith SM, Soubhi $\mathrm{H}$, Fortin $\mathrm{M}$, et al. Managing patients with multimorbidity: systematic review of interventions in primary care and community settings. BMJ 2012;345:e5205.

37. Mangin D, Heath I. Multimorbidity and Quaternary Prevention (P4). Rev Bras Med Fam Comunidade 2015;10:1.

38. Guthrie B, Payne K, Alderson P, et al. Adapting clinical guidelines to take account of multimorbidity. BMJ 2012;345:e6341-e41.

39. Britt HC, Harrison CM, Miller GC, et al. Prevalence and patterns of multimorbidity in Australia. Med J Aust 2008;189:72-7.

40. Lochner KA, Cox CS. Prevalence of multiple chronic conditions among Medicare beneficiaries, United States, 2010. Prev Chronic Dis 2013;10:E61

41. Marengoni A, Winblad B, Karp A, et al. Prevalence of chronic diseases and multimorbidity among the elderly population in Sweden. Am J Public Health 2008;98:1198-200.

42. Violan C, Foguet-Boreu Q, Hermosilla-Perez E, et al. Comparison of the information provided by electronic health records data and a population health survey to estimate prevalence of selected health conditions and multimorbidity. BMC Public Health 2013;13:251.

43. Rizza A, Kaplan V, Senn O, et al. Age- and gender-related prevalence of multimorbidity in primary care: the swiss fire project. BMC Fam Pract 2012;13:113.

44. Krieger N, Williams DR, Moss NE. Measuring social class in US public health research: concepts, methodologies, and guidelines. Annu Rev Public Health 1997;18:341-78.

45. Nagel G, Peter R, Braig S, et al. The impact of education on risk factors and the occurrence of multimorbidity in the EPIC-Heidelberg cohort. BMC Public Health 2008;8:384.

46. Violan C, Foguet-Boreu Q, Roso-Llorach A, et al. Burden of multimorbidity, socioeconomic status and use of health services across stages of life in urban areas: a cross-sectional study. BMC Public Health 2014;14:530.

47. Cortez-Dias N, Robalo Martins S, Belo A, et al. [Characterization of lipid profile in primary health care users in Portugal]. Rev Port Cardiol 2013;32:987-96.

48. Polonia J, Martins L, Pinto F, et al. Prevalence, awareness, treatment and control of hypertension and salt intake in Portugal: changes over a decade. The PHYSA study. J Hypertens 2014;32:1211-21.

49. DGS. Portugal-Saúde Mental em números-2013. Lisboa: DGS, 2013.

50. Gardete-Correia L, Boavida JM, Raposo JF, et al. First diabetes prevalence study in Portugal: PREVADIAB study. Diabet Med 2010;27:879-81.

51. do Carmo I, Dos Santos O, Camolas J, et al. Overweight and obesity in Portugal: national prevalence in 2003-2005. Obes Rev 2008:9:11-19.

52. Augusto GF. Mental health in Portugal in times of austerity. Lancet Psychiatry 2014;1:109-10.

53. Baxter AJ, Scott KM, Ferrari AJ, et al. Challenging the myth of an "epidemic" of common mental disorders: trends in the global prevalence of anxiety and depression between 1990 and 2010 . Depress Anxiety 2014;31:506-16.

54. Schafer I, von Leitner EC, Schon G, et al. Multimorbidity patterns in the elderly: a new approach of disease clustering identifies complex interrelations between chronic conditions. PLOS ONE 2010;5: e15941.

55. IDF. The IDF consensus worldwide definition of the metabolic syndrome. 2006. http://www.idf.org/webdata/docs/IDF_Meta_def_ final.pdf (accessed Aug 2015).

56. Tyrer $P$. The case for cothymia: mixed anxiety and depression as a single diagnosis. Br J Psychiatry 2001;179:191-3. 